Beyond these mechanistic aspects, the work of LeBlanc et al. has possible clinical relevance, since recombinant soluble TRAIL could soon enter clinical trials. TRAIL has displayed potent antitumor activity and low toxicity towards normal tissues in several studies in animal models ${ }^{12,13}$. Also, synergy between TRAIL and anticancer drugs has been demonstrated in diverse tumor cell types in tumor xenograft experiments. However, the results of LeBlanc et al. suggest that TRAIL is most likely to show single-agent activity in tumors that are either type I or, alternatively, type II cells where Bcl-2:Bax ratios are permissive for apoptosis. As such, patient selection for clinical trials would be benefited by availability of biomarkers that are predictive of type I versus type II status or which define the functional integrity of various apoptosis pathways. It remains, however, to be seen whether findings derived from tumor lines suffering mismatch repair defects, such as the gastrointestinal tumor lines employed for this study ${ }^{3}$, are reflective of cancer in general. More research therefore may be needed to define the optimal tumor entities for TRAIL-based regimens.

Future studies should also include a more detailed investigation into mecha- nisms of re-sensitization of Bax-deficient tumor cells by drug treatment. In this regard, agents that affect DNA methylation have sometimes proven useful for inducing re-expression of silenced pro-apoptotic genes ${ }^{14}$. Though these drugs may have been overlooked for lack of efficacy in previous trials, combination therapy with TRAIL might renew interest in this class of antineoplastic agents for solid tumors. High-density oligonucleotide/cDNA arrays could also provide a powerful tool for identifying anticancer agents that influence expression of apoptosis-relevant genes in ways that act synergistically with TRAIL.

If the results obtained in tumor xenograft studies in mice hold true when translated into the clinic for humans, then physicians may soon be hot on the trail of a new biological therapy for advanced cancers. Let's hope that concerns over potential toxicities do not deprive TRAIL of the chance to show its stuff in the clinic.

1. Reed, J.C. Apoptosis-based therapies. Nature Rev. Drug Dev. 1, 111-121 (2002).

2. Nicholson, D.W. From bench to clinic with apoptosis-based therapeutic agents. Nature 407, 810-816 (2000).

3. LeBlanc, H. et al. Tumor cell resistance to death receptor induced apoptosis through mutational inacti- vation of the proapoptotic Bcl-2 homolog Bax. Nature Med. 8, 274-281 (2002).

4. Ashkenazi, A. \& Dixit, V.M. Death receptors: signaling and modulation. Science 281, 1305-1308 (1998).

5. Salvesen, G.S. \& Dixit, V.M. Caspase activation: the induced-proximity model. Proc. Natl. Acad. Sci. USA 96, 10964-10967 (1999).

6. Ionov, Y., Yamamoto, H., Krajewski, S., Reed, J.C. \& Perucho, M. Mutational inactivation of the proapoptotic gene BAX confers selective advantage during tumor clonal evolution. Proc. Natl. Acad. Sci. USA 97, 10872-10877 (2000).

7. Scaffidi, C. et al. Two CD95 (APO-1/Fas) signaling pathways. EMBO J. 17, 1675-1687 (1998).

8. Desagher, S. et al. Bid-induced conformational change of $\mathrm{Bax}$ is responsible for mitochondrial cytochrome c release during apoptosis. J. Cell. Biol. 144, 891-901 (1999).

9. Deng, Y., Lin, Y. \& Wu X. TRAIL-induced apoptosis requires Bax-dependent mitochondrial release of Smac/DIABLO. Genes Dev. 16, 33-45 (2002).

10. Wei, M.C. et al. Proapoptotic BAX and BAK: a requisite gateway to mitochondrial dysfunction and death. Science 292, 727-730 (2001).

11. Zhang, L., Yu, J., Park, B.H., Kinzler, K.W. \& Vogelstein, B. Role of BAX in the apoptotic response to anticancer agents. Science 290, 989-992 (2000).

12. Walczak, H. et al. Tumoricidal activity of tumor necrosis factor-related apoptosis-inducing ligand in vivo. Nature Med. 5, 157-163 (1999).

13. Ashkenazi, A. et al. Safety and antitumor activity of recombinant soluble Apo2 ligand. J. Clin. Invest. 104, 155-162 (1999).

14. Soengas, M.S. et al. Inactivation of the apoptosis effector Apaf-1 in malignant melanoma. Nature 409 207-211 (2001).

The Burnham Institute,

San Diego, California, USA

Email:wroth@burnham.org or jreed@burnham.org

\title{
Telling growth receptors where to go
}

Signal transduction pathways that regulate cell proliferation are the focus of studies aimed at identifying molecular targets for anti-cancer drugs. A report in the $\mathbf{2 5}$ January issue of Cell offers a close-up look at the function of a protein necessary for the degradation of tyrosine kinase receptors; in its absence, signaling through these receptors remains switched on.

Previous work suggested that the mammalian protein hepatocyte growth factor-regulated tyrosine kinase substrate (Hrs) might be involved in endocytosis and subsequent degradation of the epidermal growth factor receptor (EGFR). Hugo J. Bellen, Thomas Lloyd and colleagues at Baylor College of Medicine cloned the Drosophila homologue of Hrs to investigate more closely the protein's role.

The two photos show immunofluorescent labeling of garland cells, which function as kidney cells in Drosophila, in wild type (A) and hrs-deficient animals (B). Lysosomes are labeled red using lysotracker, while avidin, an internalized marker for endosomes, is labeled green-yellow. Endosomes are much larger in mutant cells (B), which also have fewer and smaller lysosomes (seen as less red labeling).

The observed differences, according to the report, are due to the inability of mutant cells to invaginate endosomal membrane and form 'multi-vesicular bodies', which are more mature endosome structures containing vesicles that carry proteins to the lysosomes. In other words, hrs is required for endosomal invagination and maturation-a process necessary to separate membrane-associated proteins,
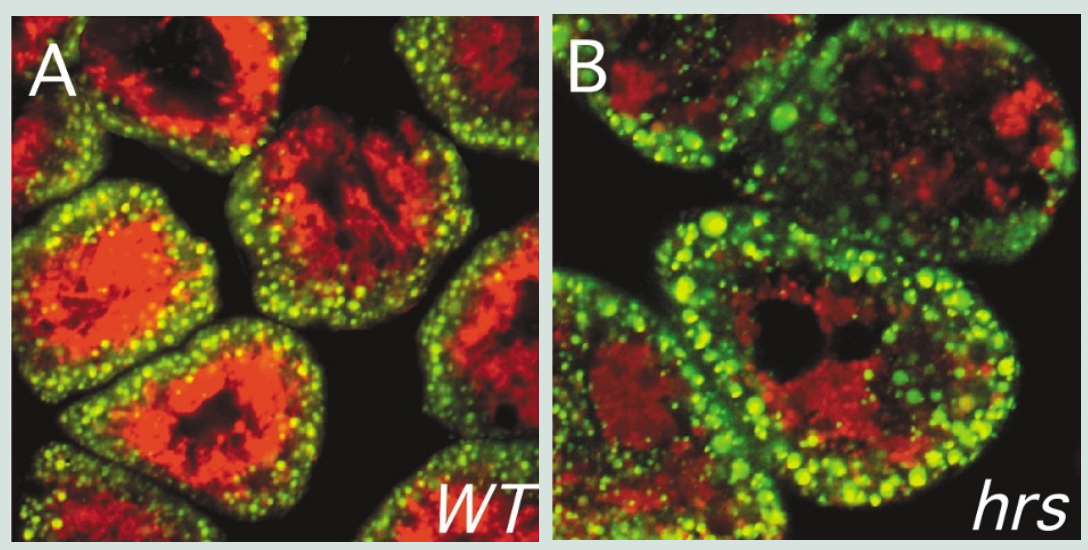
destined for degradation in the lysosomes, from those destined for recycling to the cell membrane.

In mutant flies lacking hrs and with fewer multi-vesicular bodies, EGFR and Torso tyrosine kinase receptors were not degraded, leading to enhanced tyrosine kinase signaling. If human Hrs plays a similar role, it is possible that cells lacking $\mathrm{Hrs}$ will have unregulated response to growth stimuli and become cancer cells. Indeed, another protein, TSG101, which is involved in forming multivesicular bodies like Hrs, has been shown to be deficient in some forms of cancer.

LAURA BONETTA 